\title{
Helium effects on tungsten under fusion-relevant plasma loading conditions
}

\author{
G. De Temmerman ${ }^{*}$, K. Bystrov ${ }^{\mathrm{a}}$, R.P. Doerner ${ }^{\mathrm{b}}$, L. Marot ${ }^{\mathrm{c}}$, G.M. Wright ${ }^{\mathrm{d}}$, K.B. Woller ${ }^{\mathrm{d}}$, \\ D.G. Whyte ${ }^{\mathrm{d}}$, J.J. Zielinski ${ }^{\mathrm{a}}$ \\ ${ }^{a}$ FOM Institute DIFFER, Dutch Institute For Fundamental Energy Research, Association \\ EURATOM-FOM, Trilateral Euregion Cluster, Postbus 1207, 3430BE, Nieuwegein, The Netherlands \\ ${ }^{b}$ Center for Energy Research, University of California at San Diego, 9500 Gilman Drive, La Jolla, \\ CA92093-0417, USA \\ ${ }^{c}$ Department of Physics, University of Basel, Basel, Switzerland \\ ${ }^{d}$ Plasma Science and Fusion Center, MIT, 77 Massachusetts Ave, Cambridge, 02139, USA
}

\begin{abstract}
:
Experiments were performed in the Pilot-PSI linear plasma device to study the formation of helium-induced nanostructures under high heat fluxes $\left(>10 \mathrm{MW} \cdot \mathrm{m}^{-2}\right)$, the erosion of a tungsten surface at the elevated temperatures where those structures typically form and most importantly the behaviour of a helium-induced fibreform structure during ELM-like pulses. A strong correlation between the characteristic size of the helium-induced morphology and the size of the voids observed in the near-surface region has been found, and both increase in size with increasing temperatures. Temperature-dependent erosion of tungsten surfaces was observed despite the ion energy being below the sputtering threshold, with an exponential increase of the erosion for temperatures higher than $2400^{\circ} \mathrm{C}$. The same effect was observed during ELM-like plasma pulses. Finally, a complete disappearance of the tungsten fibreform structure was observed after only one plasma pulse for energy densities higher than $0.5 \mathrm{MJ} . \mathrm{m}^{-}$ 2 .
\end{abstract}

PACS: 52.40.Hf, 52.77.Bn, 52.77.Dq

PSI-20 keywords: tungsten, helium, sputtering, tungsten fuzz 
${ }^{*}$ Corresponding author address: FOM Institute DIFFER, Dutch Institute For Fundamental Energy Research, Association EURATOM-FOM, Trilateral Euregion Cluster, Postbus 1207, 3430BE, Nieuwegein, The Netherlands

${ }^{*}$ Corresponding author e-mail: g.c.detemmerman@differ.nl

Presenting author: Gregory De Temmerman

\section{Introduction}

Tungsten is currently the plasma-facing material of choice for the ITER divertor and for future fusion devices owing to its good thermal properties and low erosion by incoming plasma particles. It is known however that bombardment of tungsten by high-fluxes of low energy particles such as helium in particular leads to strong morphology changes such as the formation of a fibreform nanostructure or the development of voids on the surface [1]. The formation conditions of such effects have been the subject of intense investigations [2, 3] and modelling efforts are ongoing to get a full physical picture of the helium trapping and interactions with the tungsten lattice [4]. The recent observation of fibreform nanostructure formation in Alcator C-mod [5], demonstrates that provided the formation conditions are met, helium-induced morphology changes are likely to occur in magnetic confinement devices such as ITER.

With that in mind, the significance of such morphology changes for the behaviour of tungsten under fusion-relevant conditions is far from clear; in particular in the divertor region where the heat and particle flux is the highest, and where ELMs are to deposit most of their energy. The main questions to address concern the formation of helium-induced nanostructures under high heat fluxes $\left(>10 \mathrm{MW} \cdot \mathrm{m}^{-2}\right)$, the erosion of a tungsten surface at the elevated temperatures where those structures typically form and most importantly the behaviour of a helium-induced fibreform structure during ELM-like pulses. Those points have been addressed by specific experiments in the Pilot-PSI linear device taking advantage of the ability to generate high heat 
fluxes and study the behaviour of refractory metals at temperatures close to the melting point both in steady-state and during transient events.

\section{Experimental}

Experiments described here were performed in the Pilot-PSI linear plasma generator, which produces plasma parameters $\left(\mathrm{n}_{\mathrm{e}}=0.1-10 \times 10^{20} \mathrm{~m}^{-3}, \mathrm{~T}_{\mathrm{e}}=0.3-5 \mathrm{eV}\right)$ relevant for the study of plasma-surface interactions in the ITER divertor. The plasma is generated by a so-called cascaded arc source and exhausts into the vacuum vessel. An axial magnetic field (0.4-1.6T) is used to confine the plasma and generate an intense magnetized cylindrical plasma beam. The maximum pulse duration is dependent on the magnetic field strength and is typically 100s at $0.4 \mathrm{~T}$ and $10 \mathrm{~s}$ at $1.6 \mathrm{~T}$. In addition, the device offers the unique possibility to combine the continuous plasma with a transient heat and particle pulse (up to $1.2 \mathrm{GW} \cdot \mathrm{m}^{-2}$ for $1 \mathrm{~ms}$ ) allowing the study of ELM effects on plasma-exposed surfaces [6, 7]. The ELM-like pulses are generated by discharging a capacitor bank system into the plasma source to transiently increase the input power and thus the plasma density and temperature. More details about the pulsed-plasma system can be found in [8]. In this paper, the source was operated in pure helium, while some experiments were done in pure argon.

The plasma parameters are measured by means of a Thomson scattering system [9] located at a distance of $17 \mathrm{~mm}$ from the plasma-exposed surface. The plasma density and temperature exhibit Gaussian profiles with a Full-Width Half Maximum (FWHM) of about $10 \mathrm{~mm}$. Plasma densities and temperature were in the range $0.4-3 \times 10^{20} \mathrm{~m}^{-3}$ and $1.3-3 \mathrm{eV}$ respectively. During the ELM-like pulse, the plasma density is in the range $1-10 \times 10^{21} \mathrm{~m}^{-3}$ while the temperature increases up to $10 \mathrm{eV}$. The ion flux to the surface is determined from the Thomson scattering measurements by assuming that ions are accelerated over the pre-sheath up to the sound speed. In addition, measurements of the ion saturation current are used to cross-check the flux values. The ion fluxes used in this study were in the range $0.7-3 \times 10^{24} \mathrm{~m}^{-}$ 


$$
{ }^{2} \mathrm{~S}^{-1}
$$

Plasma-exposed samples were made of polycrystalline tungsten cut from a rod. After polishing to a mirror finish, the samples were ultrasonically cleaned in acetone and alcohol, and outgassed at $1000^{\circ} \mathrm{C}$ for $15 \mathrm{~min}$. The samples are clamped on a water-cooled copper holder, an intermediate layer of Grafoil is used to improve the thermal contact. Note that the plasma-exposed surface has a diameter of $22 \mathrm{~mm}$, which is larger than the FWHM of the plasma beam hence the surface temperature has a Gaussian profile. A fast infrared camera (FLIR SC7500MB) is used to measure the 2D surface temperature profile. A framerate of $100 \mathrm{~Hz}$ is used during steady-state experiments while it is set to $10 \mathrm{kHz}$ for ELM-surface interaction experiments. The surface temperature is coupled with the plasma-deposited power and is controlled by varying the plasma conditions. Surface temperatures in the range 600$2400^{\circ} \mathrm{C}$ were used.

\section{Results and discussion}

\subsection{Morphology changes}

Tungsten surfaces exposed to large fluences of low energy $(<60 \mathrm{eV})$ helium ions are found to exhibit a nanostructured surface morphology consisting of nanometric filaments [2,3]. Figure 1 (a) and (b) illustrate the typical morphology observed for a surface temperature of $1000^{\circ} \mathrm{C}$, with an ion energy of $50 \mathrm{eV}$ and an exposure time of 500s. Detailed studies have been conducted to clarify the formation mechanism of such structures, which appears linked to the formation and coalescence of helium bubbles in the near-surface region inducing swelling of the surface [10]. Transmission Electron Microscopy was used to gain more insight into the inner structure of the tungsten filaments. The interference patterns between the transmitted and diffracted electron beams (fig. 1c) reveal the presence of parallel atomic planes in the filament core, indicating a crystalline structure. The identified crystallographic structure is similar to that of $\alpha$-tungsten, which is the stable form of tungsten. The existence of a 
crystalline structure contrasts with earlier observations where the filaments were assumed to have an amorphous structure [2]. Fig. 1d shows a TEM picture of tungsten filaments collected from the sample showed in fig. 1a. A large number of nanometric bubbles can be observed inside the filaments with a wide size distribution and sizes lower than $20 \mathrm{~nm}$. The voids present irregular shapes with facetted structures. No spherical voids can be observed.

A correlation between the surface temperature and the characteristic size of the heliuminduced nanostructure has recently been reported in $[11,12]$ and is further illustrated in fig. 2 . At $1000^{\circ} \mathrm{C}$, nanoscopic filaments with diameter lower than $20 \mathrm{~nm}$ (fig. 2a) are formed while the filament size increased to $100-200 \mathrm{~nm}$ at $1500^{\circ} \mathrm{C}$ (fig. 2c). In addition, the size of the voids observed in the near-surface region was observed to follow the same trend, being in the size range $10-20 \mathrm{~nm}$ at $1000{ }^{\circ} \mathrm{C}$ (fig. 2b) and up to $30-70 \mathrm{~nm}$ at $1500^{\circ} \mathrm{C}$ (fig. 2d), emphasizing the role of the helium bubbles in the observed morphology changes. When the bubble size becomes large enough, open voids are observed on the surface, as illustrated in fig. 3, rather than the fibreform structure depicted in fig. 1 . While the diameter of the tungsten fuzz is known to decrease with the fuzz length, i.e. with plasma exposure time, the size of the helium bubbles increases with time. Fig. 3 shows top views and cross-sections images of tungsten surfaces exposed at $2000^{\circ} \mathrm{C}$ for 10,30 and 1000 s respectively. This apparently different behavior can be understood by considering that the progressive development of the tungsten fibreform structure virtually limits the possibility of bubble coalescence.

\subsection{Sputtering during exposures at elevated temperatures}

The helium ion energies typically used in this study $\left(\mathrm{E}_{\mathrm{ion}}<60 \mathrm{eV}\right)$ are below the sputtering threshold of tungsten. Nonetheless, mass loss measurements revealed net erosion of the plasma-exposed samples in all cases. The net erosion yield is found to depend strongly on the incoming ion energy and surface temperature, with a factor 4 increase between 1300 and $1900^{\circ} \mathrm{C}$. While the erosion yields reported in fig. 4 a are admittedly low ( $<3 \times 10^{-4}$ at/ion), two 
points need to be kept in mind: the mass loss measurements are averaged over the sample surface which is larger than the plasma beam diameter, and it is known that under the typical plasma conditions of Pilot-PSI the net erosion yield is significantly lower than the gross erosion yield due to a large re-deposition fraction [13]. To investigate further the evolution of tungsten erosion with surface temperature, a series of experiments was done where the surface temperature was allowed to ramp up during the exposure while the WI line emission at 400.9nm was monitored. The WI line intensity is corrected for the possible blackbody radiation by line fitting and background subtraction. This is especially important in the investigated temperature range $\left(1300-2400^{\circ} \mathrm{C}\right)$. During those experiments, the electron temperature was always below $2 \mathrm{eV}$ and the electron density higher than $10^{20} \mathrm{~m}^{-3}$, in a range where no experimental measurements of the WI S/XB exist [14]. Instead of relying on extrapolations from the existing measurements of $\mathrm{S} / \mathrm{XB}$ values, the results presented here do not give absolute values of the erosion rate but rather illustrate its evolution with the experimental conditions. The plotted signals consist of the measured WI line intensity normalized by the incoming ion flux, which varies during the plasma exposure as a result of a gradual decrease of the magnetic field. As with the mass loss measurements, a strong increase of the erosion rate is observed with increasing both ion energy and surface temperature (fig. 4b). Two regimes can be distinguished. Between $1200^{\circ} \mathrm{C}$ and $2100^{\circ} \mathrm{C}$, a linear increase of the erosion yield with surface temperature is measured for 30,50 and 70ev ion energy. A similar behaviour is observed in the case of a pure argon discharge (fig. 4c) although the achievable temperature range is limited in that case. For temperatures higher than $2100^{\circ} \mathrm{C}$, an exponential increase of the sputtering yield occurs with increasing temperatures. Such conditions could only be achieved for $E_{\text {ion }}=70 \mathrm{eV}$ but are very reproducible as demonstrated in fig. $4 \mathrm{~b}$ where measurements taken for two different samples are plotted. To investigate if such behaviour would be observed with lower ion energies, experiments were done using the pulsed source 
system and a fast visible camera for simultaneous measurements of the WI line brightness (see [7] for more details). The sample was electrically floating and the electron temperature during the pulse remained below $5 \mathrm{eV}$. The temperature dependence of the WI emission is shown in fig. 5a. It is evident that a relative similarity exists between fig. 5a and fig. 4b with an exponential increase of the WI line emission with temperature after $2200^{\circ} \mathrm{C}$. Such an exponential increase is expected from the evolution of the surface evaporation rate with temperature. To compare the measured temperature dependence and what would be expected from the thermodynamic properties of tungsten and physical sputtering [15], the expected W loss rate with surface temperature is plotted in fig. 5a. The loss rate due to physical sputtering is assumed to be temperature-independent and the value measured at $900^{\circ} \mathrm{C}$ (fig. 4a) is used as a reference yield. The evaporation rate is expected to increase strongly after $3000^{\circ} \mathrm{C}$, while the experimental measurements show an exponential increase starting already at temperatures of $2200-2400^{\circ} \mathrm{C}$. A similar (but quantitative) verification of this effect was achieved using molybdenum samples. The samples were exposed to continuous helium plasmas with different surface temperatures in the range $800-2400^{\circ} \mathrm{C}$, and the net erosion yield was measured by the mass loss technique. Fig. 5b compares the experimentally measured loss rate with what would be expected assuming physical sputtering and thermal evaporation. Here also, the exponential increase of the loss rate is observed around $500^{\circ} \mathrm{C}$ below what would be expected.

Several observations of a strong increase of the erosion rate at temperatures lower than that expected from thermal evaporation have been reported in the past $[16,17]$ for both solid and liquid surfaces. The exact mechanism at stake remains unclear although some models have been proposed $[18,19]$, having in common the creation of loosely bound particles, which can be thermally released under particle bombardment. While previous observations were concentrated on low-melting point metals, the results described here confirm that a similar 
mechanism occurs for refractory metals such as molybdenum and tungsten. While the surface temperature in the ITER divertor will be well-below the point at which such effects become important, the transient thermal excursions during ELMs might increase the surface temperature enough so than enhanced material release occurs.

Chemical erosion of tungsten by oxygen is known to be temperature-dependent. For temperatures higher than $1000^{\circ} \mathrm{C}, \mathrm{WO}_{3}$ can be released by evaporation and the surface oxidation rate is expected to increase linearly with temperature up to $2250^{\circ} \mathrm{C}$ [15], which is very similar to what is measured experimentally in Pilot-PSI (fig. 4). The presence of oxygen ions (OII line at $425.4 \mathrm{~nm}$ ) is observed by spectroscopy (fig. 4d) during the helium plasma exposure. The OII line intensity, measured close to the sample surface, increases strongly with the surface temperature between 1200 and $2000^{\circ} \mathrm{C}$ although no clear evolution of the OII signal with ion energy could be observed. Similar increase of both the WI and OII line intensities with temperatures were reported during exposure of a tungsten-coated graphite limiter in TEXTOR [20]. The presence of a low fraction of oxygen ions in the plasma cannot be excluded, and given the high ion flux in Pilot-PSI, even low amounts of oxygen in the plasma would represent a high absolute oxygen flux (in the range of $10^{21} \mathrm{~m}^{-2} \mathrm{~s}^{-1}$ for $\mathrm{c}_{\mathrm{O}}<0.1 \%$ ) The chemical sputtering of tungsten by low energy oxygen ions $(<100 \mathrm{eV})$ is not well documented in the literature. In [21], sputtering yields of 0.07-0.08 were measured for oxygen ion energies of $150 \mathrm{eV}$ in the range $800-1600^{\circ} \mathrm{C}$. Assuming a similar yield for oxygen ion energy of $50 \mathrm{eV}$ and $\mathrm{c}_{0}=0.1 \%$ in the plasma would translate into an erosion yield $7-8 \times 10^{-5}$ while the measured yield (fig. $4 a$ ) is in the range $5 \times 10^{-5}-3 \times 10^{-4}$. Chemical erosion by traces amount of oxygen ions would therefore explain the observed temperature dependence in the range $900-2100^{\circ} \mathrm{C}$.

\subsection{Effect of ELM-like pulses on helium-induced morphology}

Tungsten samples were exposed in the PISCES-A linear plasma device to pure helium 
plasmas in order to create a homogeneous layer of tungsten 'fuzz' over the surface with a thickness of about $2 \mu \mathrm{m}$. Those samples were subsequently exposed in Pilot-PSI to an ELMlike plasma pulse superimposed on a pure helium plasma. The plasma duration was set to $4 \mathrm{~s}$ with a magnetic field of $1.6 \mathrm{~T}$, the ELM-like pulse occurred at $\mathrm{t}=2 \mathrm{~s}$ and the peak energy density was $0.5 \mathrm{MJ} . \mathrm{m}^{-2}$. The heat flux profile was Gaussian with a FWHM of about $10 \mathrm{~mm}$ so that different areas of the surface are exposed to different peak heat loads. The surface temperature was followed by a fast infrared camera (framerate of $10 \mathrm{kHz}$ ) through the exposure. The determination of the exact surface temperature is however made very difficult by the very different emissivity of the 'fuzzy' surface (which appears black [22]) and that of the original tungsten substrate. Fig. 6 shows the measured time evolution of the surface temperature for two different assumptions on the surface emissivity: either a fixed emissivity of 0.8 representing that of the black helium-modified surface, or the emissivity of polished tungsten taking into account the temperature-dependence of the emissivity [15]. Before the plasma pulse, the surface appears un-modified (form the IR view) and the surface temperature is around $400^{\circ} \mathrm{C}$. After only 1 sequence (4s continuous plasma +1 pulse), the surface is completely modified with the target centre, exposed to the highest heat flux, appearing as shiny as the outer rim which was not covered with fuzz (inset of fig. 7). High-resolution microscopy analyses were done at several locations across the surface (fig. 7). Since the heat flux deposition profile is known, a correlation between the location on the surface and the absorbed energy density is possible. For energy densities below $0.1 \mathrm{MJ} . \mathrm{m}^{-2}$ (fig. $7 \mathrm{a}$ ), the fuzz structure appears totally undamaged. A gradual disappearance of the fuzz structure is observed for higher energy densities. At $0.25 \mathrm{MJ}_{\mathrm{m}} \mathrm{m}^{-2}$, the characteristic size of the structure increases up to $200-300 \mathrm{~nm}$ (fig. 7b). Increasing the absorbed energy to $0.35 \mathrm{MJ} \cdot \mathrm{m}^{-2}$ leaves the surface with some micron-scale roughness (fig. 7c), while some voids can be observed at sparse locations on the surface. Finally, in the region exposed to the highest heat load (fig. 
7d), large tungsten grains can be observed with no trace of any helium-induced surface morphology changes remaining on the surface. This observation is confirmed by cross-section analysis of the sample as illustrated in fig. 8, where the cross-section of a typical heliummodified surface is compared with that of the sample exposed to the ELM-like pulse. Except the obvious disappearance of the initial structure, the striation observed in fig. $8 \mathrm{~b}$ is the only indication that some changes might have occurred in the material. Repeat of the experiment yielded a similar observation of the disappearance of the fuzz structure and rendered experiments with higher number of ELM-like pulses difficult. No trace of arcing nor melting was observed anywhere on the surface. Those observations contrast with those reported in [23] where the helium-induced nanostructure was found to withstand plasma pulses created with a plasma gun with minimum surface modifications. On the other hand, the surface morphology obtained for a pulse energy of $0.35 \mathrm{MJ}^{-\mathrm{m}^{-2}}$ has some resemblances with that described in [24] when the surface temperature of a helium-plasma exposed tungsten target was increased for short periods of times (1-20min) by positive biasing of the sample. Furthermore, annealing of a fully developed fuzz structure at $1630^{\circ} \mathrm{C}$ lead to the disappearance of the helium nanostructure. In those two cases, no material loss was observed suggesting that evaporation or melting of the surface did not happen. It is worth pointing out that the cross section of the sample after annealing in [25] is very similar to that of fig.7b with the presence of striations below the surface. It is also interesting to note that desorption peaks of helium were found at 770,1170 and $1530^{\circ} \mathrm{C}$ and complete disappearance of the structure is observed when the surface temperature is higher than the desorption temperature. Admittedly, the latter two experiments are made on timescales much longer than in the present experiments. However, the temperature during an ELM-like pulse being much higher could accelerate the re-organization of the surface following desorption of the trapped helium particles. The similarities between those observations point out the fact that the helium- 
induced nanostructure can be annealed out once helium is able to escape the surface, and would indicate that the presence of sufficiently large ELMs will prevent the formation of helium-induced structures.

\section{Summary and conclusion}

The behaviour of tungsten surfaces exposed to helium plasmas under fusion-relevant plasma conditions at elevated temperatures has been investigated in the Pilot-PSI linear plasma device. Helium-induced morphology changes are found to be mainly driven by the development of helium bubbles in the near-surface region. The size of the near-surface voids increases with the surface temperature during exposure, as the characteristic size of the observed morphology does.

During those experiments, erosion of the tungsten surfaces was measured both by mass loss and optical spectroscopy despite the ion energy being lower than the sputtering threshold. The erosion rate increases linearly with temperature in the range $1200^{\circ} \mathrm{C}$ and $2100^{\circ} \mathrm{C}$ and increases exponentially for higher temperatures. It was demonstrated that the enhanced material loss at elevated temperatures observed for metals such as Be or Li also occurs for refractory metals. This needs to be considered as a possible channel for tungsten erosion in ITER during ELMs. Finally, initial experiments revealed that the helium-induced nanostructure formation did not pose issues in terms of dust formation during ELM-like pulses. Instead, the observed complete disappearance of the nanostructure for energy densities higher than $0.5 \mathrm{MJ} . \mathrm{m}^{-2}$ would indicate that the formation of such a structure would be prevented by large ELMs.

\section{Acknowledgements}

The authors gratefully acknowledge the help and support from the Pilot-PSI staff. This work is part of the research program of the Stichting voor Fundamenteel Onderzoek der Materie (FOM), which is financially supported by the Nederlandse 
Organisatie voor Wetenschappelijk Onderzoek (NWO). It is supported by the European Communities under the contract of Association between EURATOM and FOM and carried out within the framework of the European Fusion Program, and of the European Taskforce on Plasma-Wall Interactions.

\section{References:}

[1] Y. Ueda, J. Nucl. Mater., in press

[2] M.J. Baldwin, and R.P. Doerner, Nucl. Fusion 48 (2008) 035001

[3] S. Kajita, et al, Nucl. Fusion, 49 (2009) 095005

[4] Y.G. Li, et al, Commun. Comput. Phys., 11 (2012) 1547

[5] G.M. Wright, et al, these proceedings

[6] G. De Temmerman et al, Appl. Phys. Lett., 97 (2010) 081502

[7] G. De Temmerman et al, Nucl. Fusion, 51 (2011) 073008

[8] J.J. Zielinski, Plasma Sources Sci. Techn., submitted

[9] H.J. van der Meiden, Rev. Sci. Instrum., 79 (2008) 013505

[10] S.I. Krasheninnikov, Phys. Scr. T145, (2011) 014040

[11] G. De Temmerman et al, J. Vac. Sci. Tech. A, submitted

[12] S. Kajita et al, J. Nucl. Mater., 418 (2011) 152

[13] K. Bystrov et al, J. Nucl. Mater., 415 (2011) S149

[14] D. Nishijima, et al, Phy. Plasmas 18 (2011) 019901

[15] 'Tungsten: Properties, Chemistry, Technology of the Element, Alloys, and Chemical Compounds’, Kluwer Academic 2010

[16] R.W. Conn et al, Nucl. Fusion 42 (2002) 1060

[17] R.P. Doerner, et al, J. Nucl. Mater., 290-293 (2001) 166 
[18] R.P. Doerner, S.I. Krasheninnikov, and K. Schmid, J. Appl. Phys., 95 (2004) 4471

[19] J.P. Allain, M.D. Coventry, and D.N. Ruzic, Phys. Rev. B, 76 (2007) 205434

[20] M. Psoda, et al, J. Nucl. Mat., 386-388 (2009) 740

[21] E. Hechtl, W. Eckstein, J. Roth, and J. Laszlo, J. Nucl. Mater., 179-181 (1991) 290

[22] S. Kajita, et al, Appl. Phys. Express, 3 (2010) 085204

[23] D. Nishijima, et al, Fus. Sci. Technol., 60 (2011) 1447

[24] S. Takamura, T. Miyamoto, Y. Yomida, T. Minagawa, N. Ohno, J. Nucl. Mater. 415 (2011) S100

[25] M.J. Baldwin, et al, Journal of Nuclear Materials 404 (2010) 165-173 
Figure 1: Characteristics of the fibreform nano-structure formed under irradiation of a tungsten surface at $1000^{\circ} \mathrm{C}$ by low-energy helium ions (50eV) for a duration of 500s. (a) and (b) top-view and cross-section images. (c) Electron diffraction patter from the tungsten filaments of (a). (d) High-resolution TEM image of the structure of a tungsten filament

Figure 2: Top view and cross-section images of a helium-modified tungsten surface exposed at $1500^{\circ} \mathrm{C}$ and $1000^{\circ} \mathrm{C}$ for 60 s with $\mathrm{E}_{\mathrm{ion}}=40 \mathrm{eV}$

Figure 3: Evolution of the helium bubble size as a function of the exposure time for a surface temperature of $2000^{\circ} \mathrm{C}$ and $\mathrm{E}_{\mathrm{ion}}=40 \mathrm{eV}$. Top views and cross-sections are shown on the left and right panel respectively. $t=10$ s (a and b), $t=30$ s (c and $d$ ) and $t=1000$ s (e and f)

Figure 4: (a) net erosion yield of tungsten bombarded by helium ions as a function of surface termpature. (b) and (c) evolution of the gross erosion (WI line intensity normalized to ion flux) of tungsten as a function of temperature for helium (b) and argon (b) ions. (d) Evolution of the OII normalized intensity as a function of temperature and helium ion energy.

Figure 5: (a) Evolution of the WI line brigthness as a function of the surface temperature during an ELM-like plasma pulse in Pilot-PSI. Also plotted is the expected evolution of the tungsten loss rate assuming a temperature-independent sputtering and thermal evaporation. (b) Comparison of the measured Mo loss rate as a function of temperature during exposure to $50 \mathrm{eV}$ helium ions and of the expected loss rate from sputtering and evaporation. 
Figure 6: Evolution of the surface temperature of a helium-modified tunsgsten surface during a plasma pulse in Pilot-PSI assuming two cases for the surface emissivity

Figure 7: Evolution of the morphology of a 'fuzzy' tungsten surface as a function of the absorbed energy density during ELM-simulation experiments in Pilot-PSI

Figure 8: Comparison of the cross-sections of a tungsten surface covered with a fibreform structure formed in PISCES-A before (a) and after (b) exposure to an ELM-like pulse in PilotPSI 
Figure 1:

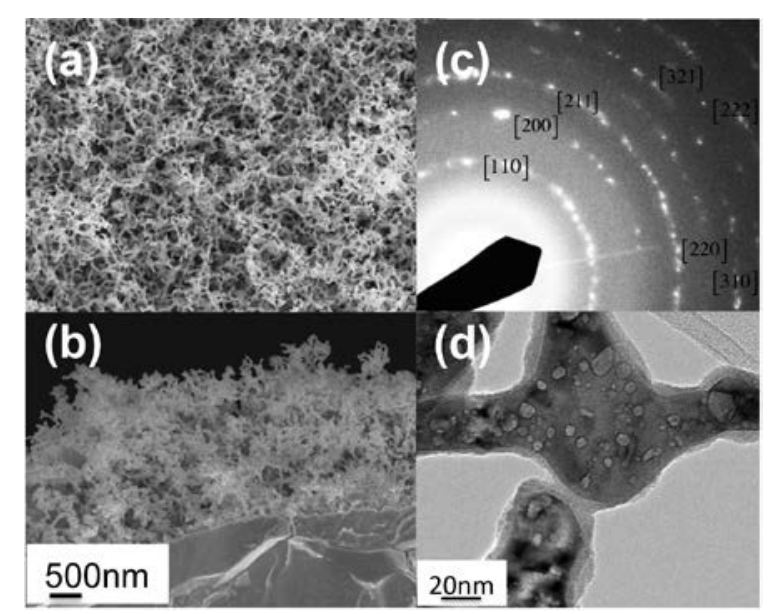


Figure 2:

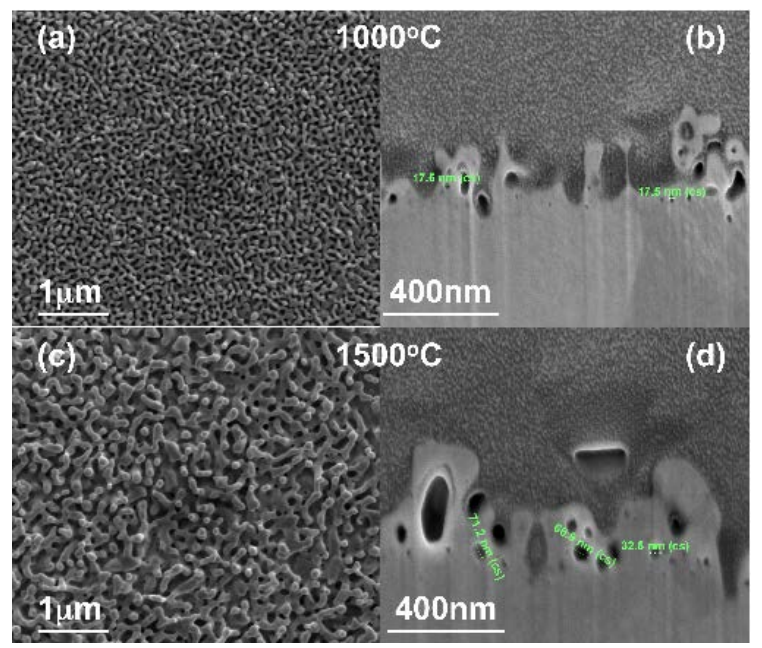


Figure 3:

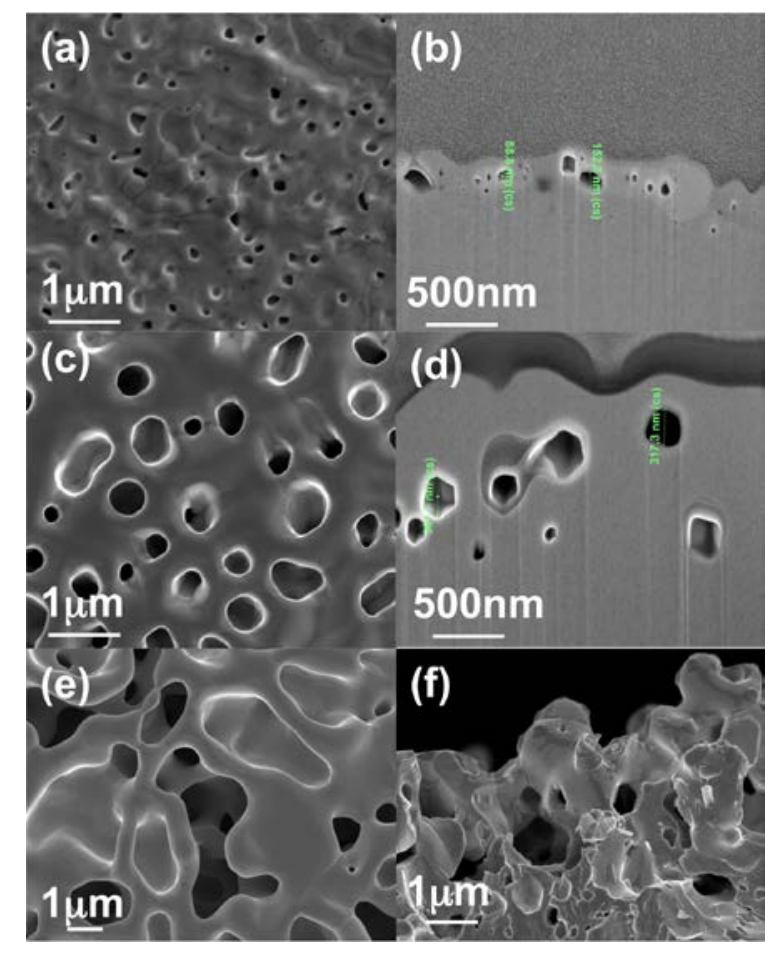


Figure 4:
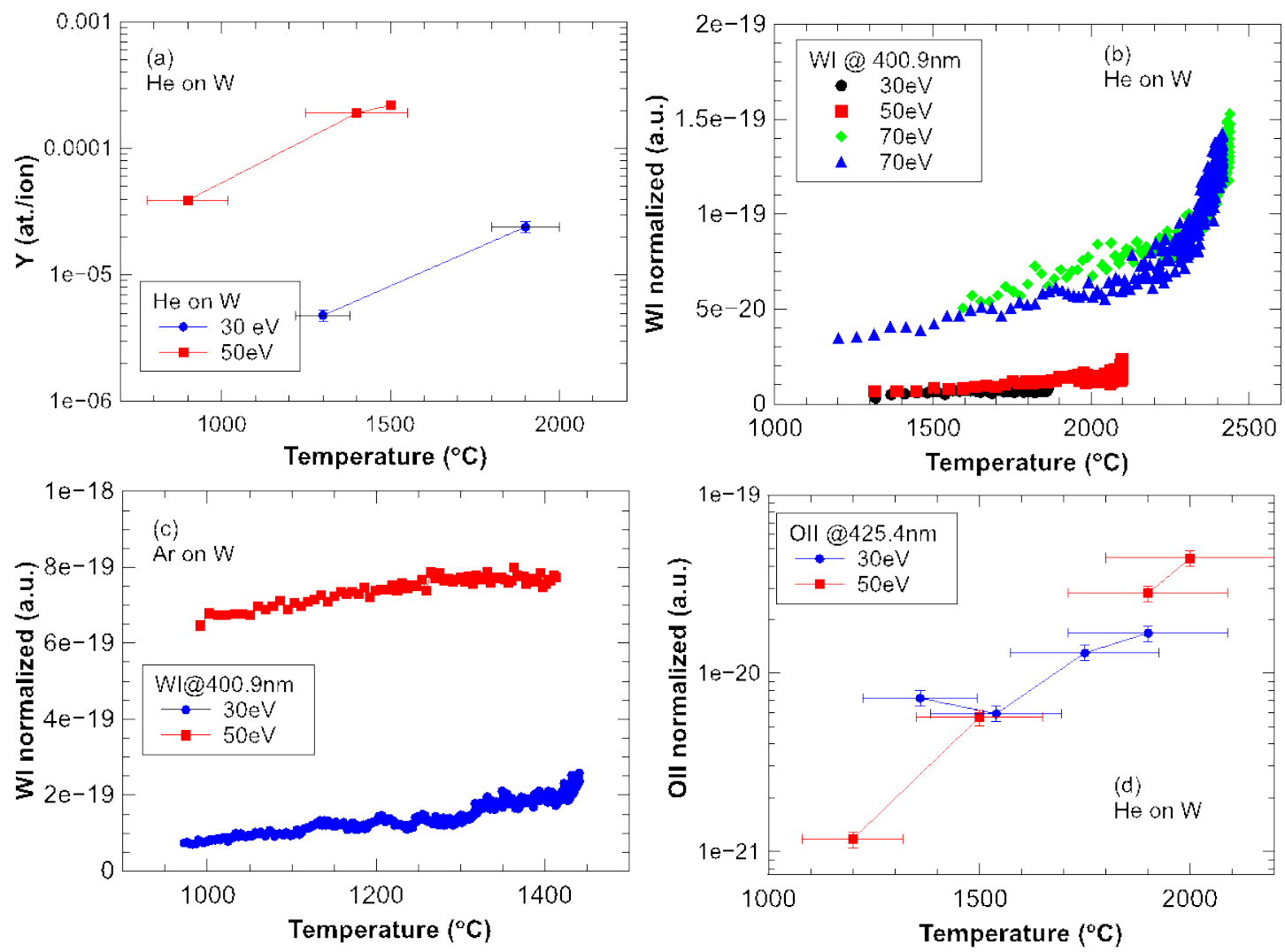
Figure 5:
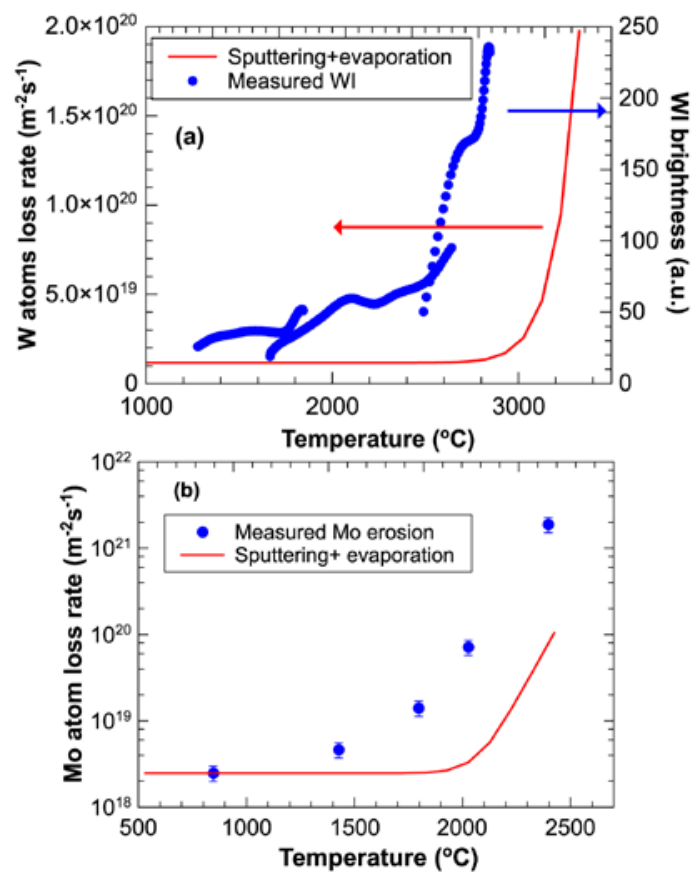
Figure 6:

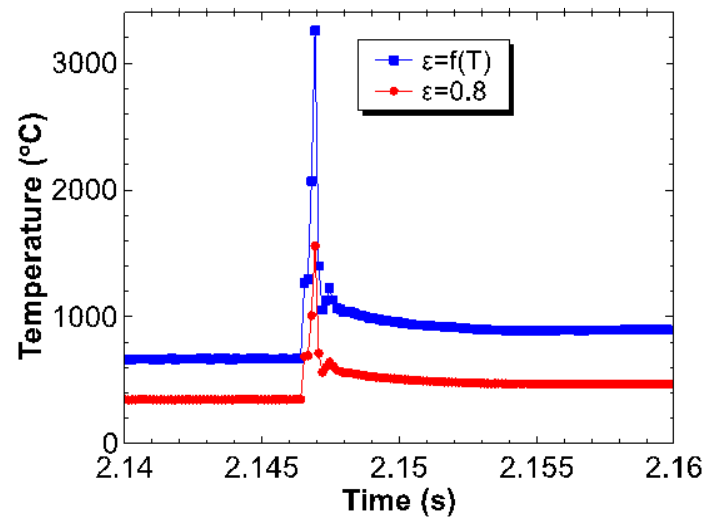


Figure 7:

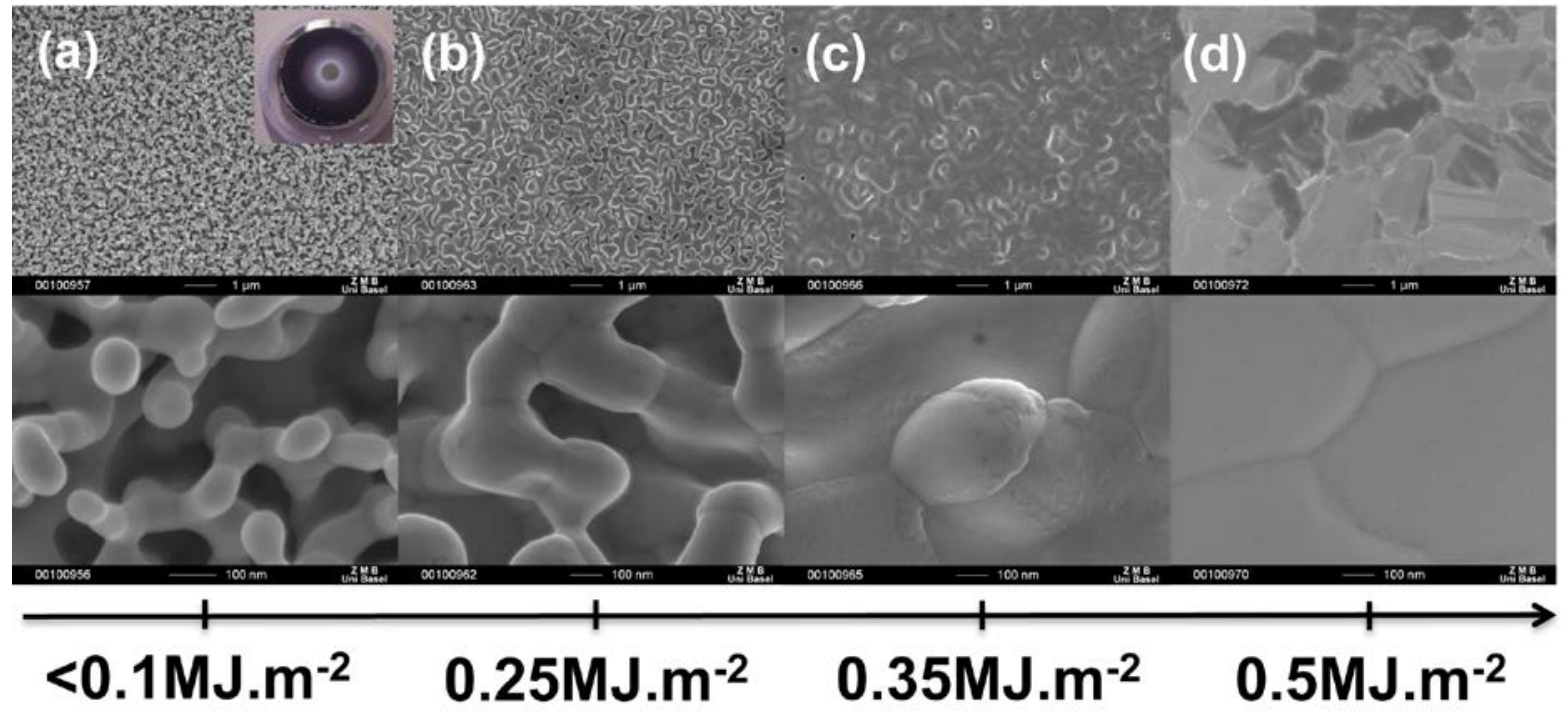


Figure 8:

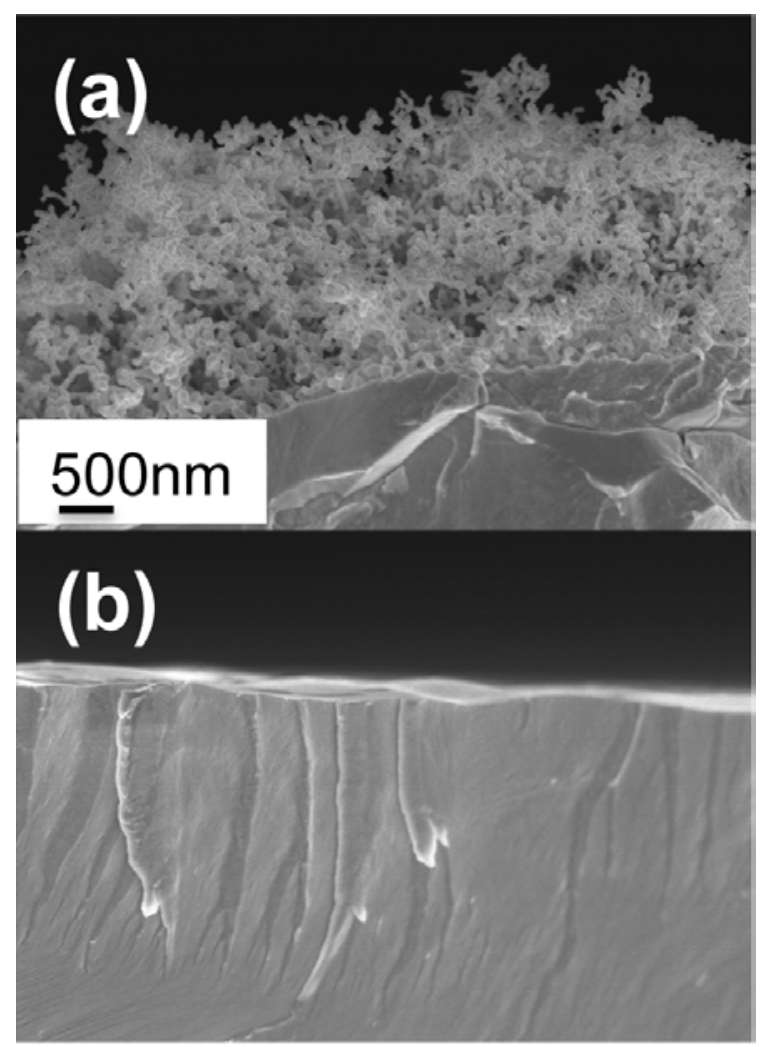

